Algebra and its Applications

Elsevier Editorial System(tm) for Linear

Manuscript Draft

Manuscript Number:

Title: Spectra, signless Laplacian and Laplacian spectra of complementary prisms of graphs

Article Type: Regular Issue

Keywords: Graph operations; complementary prism of a graph; graph eigenvalues

Corresponding Author: Dr. Domingos Moreira Cardoso, Ph.D.

Corresponding Author's Institution: University of Aveiro

First Author: Domingos Moreira Cardoso, Ph.D.

Order of Authors: Domingos Moreira Cardoso, Ph.D.; Paula Carvalho, Ph.D.; Maria A Freitas, Ph.D.; Cybele T Vinagre, Ph.D. 


\title{
Spectra, signless Laplacian and Laplacian spectra of complementary prisms of graphs
}

\author{
Domingos M. Cardoso \\ Paula Carvalho \\ Maria Aguieiras A. de Freitas \\ Cybele T. M. Vinagre
}

August 3, 2017

\begin{abstract}
The complementary prism $G \bar{G}$ of a graph $G$ is obtained from the disjoint union of $G$ and its complement $\bar{G}$ by adding an edge for each pair of vertices $\left(v, v^{\prime}\right)$, where $v$ is in $G$ and its copy $v^{\prime}$ is in $\bar{G}$. The Petersen graph $C_{5} \overline{C_{5}}$ and, for $n \geq 2$, the corona product of $K_{n}$ and $K_{1}$ which is $K_{n} \overline{K_{n}}$ are examples of complementary prisms. This paper is devoted to the computation of eigenpairs of the adjacency, the signless Laplacian and the Laplacian matrices of a complementary prism $G \bar{G}$ in terms of the eigenpairs of the corresponding matrices of $G$. Particular attention is given to the complementary prisms of regular graphs. Furthermore, Petersen graph is shown to be the unique complementary prism which is a strongly regular graph.
\end{abstract}

AMS Subject Classification: 05C50, 05C76, $15 \mathrm{~A} 18$.

Keywords: Graph operations; complementary prism of a graph; graph eigenvalues.

\section{Introduction}

In [8], Haynes et al. introduced the complementary product of two graphs, a generalization of the cartesian product, and dedicated particular attention to the special case of complementary prism of a graph, which can be seen as a variant of a prism, the cartesian product of a cycle by $K_{2}$. In precise terms, the complementary prism of a graph $G$ with complement graph $\bar{G}$, is the graph denoted by $G \bar{G}$ such that $V(G \bar{G})=V(G \cup \bar{G})$ and for which the edges are those in $G$ and $\bar{G}$ together with the edges connecting each vertex of $G$ with its copy in $\bar{G}$. Examples of complementary prisms are the Petersen graph $C_{5} \overline{C_{5}}$ and the corona product $K_{n} \circ K_{1}$ of the complete graph and $K_{1}$, which is $K_{n} \overline{K_{n}}$. Figure 1 depicts the complementary prism of $G=K_{2}+K_{1}$.

From now on, the vertices of $G \bar{G}$ in its part $G$ are denoted by letters or numbers and correspondent vertices in $\bar{G}$ by letters or numbers with apostrophe. Taking into account that if a graph is not connected, then its complement is connected, it follows that the complementary prism $G \bar{G}$ of every graph $G$ is a connected graph.

Several combinatorial properties of complementary prisms of graphs have been obtained in the literature. In [8], the diameter of $G \bar{G}$ is proved to be 


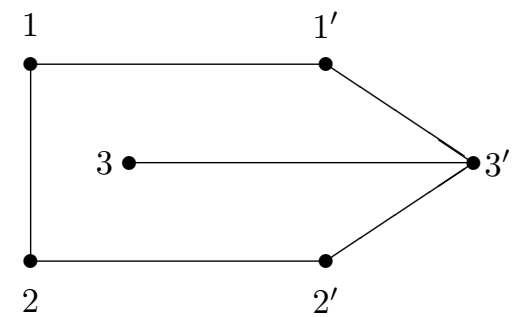

Figure 1: The complementary prism $G \bar{G}$ of the graph $G=K_{2}+K_{1}$.

not greater than 3 for every graph $G$, besides results concerning degrees, distances, independence and domination numbers and a list of questions and open problems. In [9], among other results, the characterizations of graphs $G$ whose complementary prisms attain lower bounds on domination and total domination numbers introduced in [8], were given. In [6] the authors studied algorithmic/complexity properties of complementary prisms of graphs, regarding cliques, independent sets, $k$-domination and $P_{3}$-convexity. An efficient characterization of the circumference of the complementary prism of a tree is presented in [12], where it is also shown that the Hamiltonicity of $G \bar{G}$ for graphs $G$ of bounded maximum degrees can be decided in polynomial time. However, as we know, regarding the spectra of matrices associated to complementary prisms of graphs so far there are no published results. The main goal of the current paper is the determination of the spectra, signless Laplacian and Laplacian spectra of complementary prisms of graphs.

The graphs $G$ considered in this work are simple and undirected with order (number of vertices) $n$. The vertex set of $G$ is denoted by $V(G)$ and its edge set by $E(G)$. The adjacency matrix of $G$ is the $n \times n$ matrix $\mathbf{A}_{G}$ whose $(i, j)$-entry is equal to 1 whether $i j \in E(G)$, that is, if the vertices $i$ and $j$ are adjacent, and is equal to 0 otherwise. The signless Laplacian (Laplacian) matrix of $G$ is the matrix $\mathbf{Q}_{G}=\mathbf{D}_{G}+\mathbf{A}_{G}\left(\mathbf{L}_{G}=\mathbf{D}_{G}-\mathbf{A}_{G}\right)$, where $\mathbf{D}_{G}=\operatorname{diag}\left(d_{1}, \cdots, d_{n}\right)$ is the diagonal matrix of the vertex degrees of $G$. The matrices $\mathbf{A}_{G}, \mathbf{Q}_{G}$ and $\mathbf{L}_{G}$ are symmetric and then they have $n$ real eigenvalues which are herein indexed in non increasing order according to the following notation: $\lambda_{1} \geq \lambda_{2} \geq \cdots \geq \lambda_{n}$ are the $n$ eigenvalues of $\mathbf{A}_{G}$ (also called the eigenvalues of $G$ ); $q_{1} \geq q_{2} \geq \cdots \geq q_{n}$ are the $n$ eigenvalues of $\mathbf{Q}_{G}$ and $\mu_{1} \geq \mu_{2} \geq \cdots \geq \mu_{n}$ are the $n$ eigenvalues of $\mathbf{L}_{G}$. Notice that $\mu_{n}(G)=0$ (the all one vector $\mathbf{j}$ is an associated eigenvector) and its multiplicity is equal to the number of components of $G$. For convenience, we shall sometimes denote by $\mathbf{M}_{G}$ a matrix representing the graph $G$ which can be $\mathbf{A}_{G}, \mathbf{Q}_{G}$ or $\mathbf{L}_{G}$. Then the multiset (that is, the set with possible repetitions) of eigenvalues of $\mathbf{M}_{G}$ is called the $\mathbf{M}_{G^{-}}$spectrum and denoted by $\sigma_{\mathbf{M}}(G)$ (particularly, the $\mathbf{A}_{G}$-spectrum is usually called the spectrum of $G$ ). Assuming that $\mathbf{M}_{G}$ has $p$ distinct eigenvalues, we write $\sigma_{\mathbf{M}}(G)=\left\{\gamma_{1}^{\left[r_{1}\right]}, \ldots, \gamma_{p}^{\left[r_{p}\right]}\right\}$, where the exponents indicate the multiplicity of the corresponding eigenvalue. If $\rho$ is a $\mathbf{M}_{G}$-eigenvalue and $\mathbf{u}$ is an associated eigenvector, then $(\rho, \mathbf{u})$ is called an eigenpair of $\mathbf{M}_{G}$. A $\mathbf{M}_{G}$-eigenvalue $\rho$ of $G$ is said to be a main eigenvalue if there is an associated eigenvector $\mathbf{u}$ which is not orthogonal to $\mathbf{j}$. Otherwise, we say that $\rho$ is non-main. Further concepts not herein defined can be found in 
$[2,3,4]$.

This paper is organized as follows. Section 2 is devoted to a general approach to M-eigenvalues of complementary prisms as a nonlinear eigenvalue problem. In Section 3, all the A-eigenpairs of $G \bar{G}$ but two for each main eigenvalue of $G$ are determined. Particular attention is given to the complementary prism of a connected regular graph $G$, whose spectrum is straighforwardly determined in terms of the eigenvalues of $G$. Furthermore, we prove that the Petersen graph $\left(C_{5} \overline{C_{5}}\right)$ is the unique complementary prism which is a strongly regular graph. In Section 4 the results obtained in Section 3 for the adjacency matrix are extended and updated for the signless Laplacian matrix. In Section 4 the Laplacian spectrum of the complementary prisms $G \bar{G}$ is completely determined in terms of the order and Laplacian eigenvalues of $G$. The paper finishes with conclusions and some open problems.

\section{M-characteristic polynomial of the complemen- tary prism of a graph}

As before, let us use $\mathbf{M}_{G}$ to denote the adjacency, the signless Laplacian or the Laplacian matrix of $G$. Then the matrix representing the complementary prism $G \bar{G}$ of $G$ is defined as follows:

$$
\mathbf{M}_{G \bar{G}}=\left(\begin{array}{cc}
\mathbf{M}_{G}+\delta \mathbf{I} & \tau \mathbf{I} \\
\tau \mathbf{I} & \mathbf{M}_{\bar{G}}+\delta \mathbf{I}
\end{array}\right),
$$

where $\mathbf{M} \in\{\mathbf{A}, \mathbf{Q}, \mathbf{L}\}, \quad \delta=\left\{\begin{array}{ll}0, & \text { if } \mathbf{M}=\mathbf{A} \\ 1, & \text { otherwise }\end{array}, \quad \tau=\left\{\begin{aligned}-1, & \text { if } \mathbf{M}=\mathbf{L} \\ 1, & \text { otherwise }\end{aligned}\right.\right.$ and I is the identity matrix. Let $\left(\begin{array}{r}\mathbf{u} \\ -\mathbf{v}\end{array}\right)$ be an eigenvector associated to the eigenvalue $\rho$ of $\mathbf{M}_{G \bar{G}}$. Then, from the eigenvector equation, $\mathbf{M}_{G \bar{G}}\left(\begin{array}{r}\mathbf{u} \\ -\mathbf{v}\end{array}\right)=$ $\rho\left(\begin{array}{r}\mathbf{u} \\ -\mathbf{v}\end{array}\right)$, it is immediate that $\mathbf{u} \neq \mathbf{0} \neq \mathbf{v}$. Therefore,

$$
\begin{aligned}
\mathbf{M}_{G \bar{G}}\left(\begin{array}{r}
\mathbf{u} \\
-\mathbf{v}
\end{array}\right)=\rho\left(\begin{array}{r}
\mathbf{u} \\
-\mathbf{v}
\end{array}\right) & \Leftrightarrow\left\{\begin{array}{rr}
\left(\mathbf{M}_{G}+\delta \mathbf{I}\right) \mathbf{u}-\tau \mathbf{v}= & \rho \mathbf{u} \\
\tau \mathbf{u}-\left(\mathbf{M}_{\bar{G}}+\delta \mathbf{I}\right) \mathbf{v}= & -\rho \mathbf{v}
\end{array}\right. \\
& \Leftrightarrow\left\{\begin{aligned}
\left(\mathbf{M}_{G}-(\rho-\delta) \mathbf{I}\right) \mathbf{u}= & \tau \mathbf{v} \\
\left(\mathbf{M}_{\bar{G}}-(\rho-\delta) \mathbf{I}\right) \mathbf{v}= & =\mathbf{u}
\end{aligned}\right.
\end{aligned}
$$

and thus

$$
\begin{aligned}
& \left(\mathbf{M}_{G}-(\rho-\delta) \mathbf{I}\right)\left(\mathbf{M}_{\bar{G}}-(\rho-\delta) \mathbf{I}\right) \mathbf{v}=\mathbf{v} \\
& \left(\mathbf{M}_{\bar{G}}-(\rho-\delta) \mathbf{I}\right)\left(\mathbf{M}_{G}-(\rho-\delta) \mathbf{I}\right) \mathbf{u}=\mathbf{u}
\end{aligned}
$$

The matrices $\left(\mathbf{M}_{G}-(\rho-\delta) \mathbf{I}\right)\left(\mathbf{M}_{\bar{G}}-(\rho-\delta) \mathbf{I}\right)$ and $\left(\mathbf{M}_{\bar{G}}-(\rho-\delta) \mathbf{I}\right)\left(\mathbf{M}_{G}-(\rho-\right.$ $\delta) \mathbf{I}$ ) have the same spectrum (see, for instance, [13, Th. 3.24]). Now, we have the following result.

Theorem 2.1. Let $G$ be a graph of order $n$. Then $\rho$ is an eigenvalue of $\mathbf{M}_{G \bar{G}}$ if and only if 1 is an eigenvalue of the matrix $\left(\mathbf{M}_{G}-(\rho-\delta) \mathbf{I}\right)\left(\mathbf{M}_{\bar{G}}-(\rho-\delta) \mathbf{I}\right)$. 
Additionally, if $\mathbf{v}$ and $\mathbf{u}$ are the associated eigenvectors obtained in (3) and (4), respectively, then $\left(\begin{array}{r}\mathbf{u} \\ -\mathbf{v}\end{array}\right)$ is an eigenvector of $\mathbf{M}_{G \bar{G}}$ associated to $\rho$.

Proof. Let $\rho$ be an eigenvalue of $\mathbf{M}_{G \bar{G}}$. Then, from (2) we obtain (3) (and also $(4))$ and thus 1 is an eigenvalue of the matrix $\left(\mathbf{M}_{G}-(\rho-\delta) \mathbf{I}\right)\left(\mathbf{M}_{\bar{G}}-(\rho-\delta) \mathbf{I}\right)$. Conversely, let us assume that 1 is an eigenvalue of the matrix $\left(\mathbf{M}_{G}-(\rho-\right.$ $\delta) \mathbf{I})\left(\mathbf{M}_{\bar{G}}-(\rho-\delta) \mathbf{I}\right)$. Then, there exists $\mathbf{v} \neq \mathbf{0}$ such that $\left(\mathbf{M}_{G}-(\rho-\delta) \mathbf{I}\right)\left(\mathbf{M}_{\bar{G}}-\right.$ $(\rho-\delta) \mathbf{I}) \mathbf{v}=\mathbf{v}$. Setting $\left(\mathbf{M}_{\bar{G}}-(\rho-\delta) \mathbf{I}\right) \mathbf{v}=\tau \mathbf{u}$, we obtain $(2)$ and thus $\left(\begin{array}{r}\mathbf{u} \\ -\mathbf{v}\end{array}\right)$ is an eigenvector of $\mathbf{M}_{G \bar{G}}$ associated to $\rho$.

As immediate consequence of Theorem 2.1, we have the following corollary.

Corollary 2.2. The eigenvalues of $\mathbf{M}_{G \bar{G}}$ are the roots of the polynomial

$$
p(\rho)=\operatorname{det}\left(\left(\mathbf{M}_{G}-(\rho-\delta) \mathbf{I}\right)\left(\mathbf{M}_{\bar{G}}-(\rho-\delta) \mathbf{I}\right)-\mathbf{I}\right),
$$

that is, $\operatorname{det}\left(\mathbf{M}_{G \bar{G}}-\rho \mathbf{I}\right)=\operatorname{det}\left(\left(\mathbf{M}_{G}-(\rho-\delta) \mathbf{I}\right)\left(\mathbf{M}_{\bar{G}}-(\rho-\delta) \mathbf{I}\right)-\mathbf{I}\right)$. More particularly, the roots of the polynomials

$$
\begin{aligned}
& \text { 1. } p_{\mathbf{A}}(\lambda)=\operatorname{det}\left(\left(\mathbf{A}_{G}-\lambda \mathbf{I}\right)\left(\mathbf{A}_{\bar{G}}-\lambda \mathbf{I}\right)-\mathbf{I}\right), \\
& \text { 2. } p_{\mathbf{Q}}(q)=\operatorname{det}\left(\left(\mathbf{Q}_{G}-(q-1) \mathbf{I}\right)\left(\mathbf{Q}_{\bar{G}}-(q-1) \mathbf{I}\right)-\mathbf{I}\right) \text {, } \\
& \text { 3. } p_{\mathbf{L}}(\mu)=\operatorname{det}\left(\left(\mathbf{L}_{G}-(\mu-1) \mathbf{I}\right)\left(\mathbf{L}_{\bar{G}}-(\mu-1) \mathbf{I}\right)-\mathbf{I}\right) \text {, }
\end{aligned}
$$

are the eigenvalues of the matrices $\mathbf{A}_{G \bar{G}}, \mathbf{Q}_{G \bar{G}}$ and $\mathbf{L}_{G \bar{G}}$, respectively.

Example 2.3. Applying Corollary 2.2 to the graph $G$ depicted in Figure 2, the obtained spectrum, signless Laplacian spectrum and Laplacian spectrum (the roots of the above polynomials) of $G \bar{G}$ are the following.

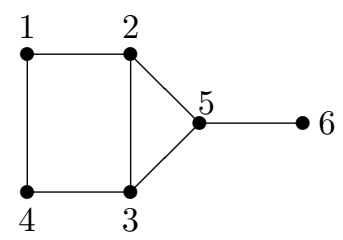

Figure 2: Graph $G$.

$$
\begin{aligned}
\sigma_{\mathbf{A}}(G \bar{G})= & \{3.62345,1.93294,1.30278,1.30278,0.930229,0.618034,0.3001, \\
& -1.618034,-1.82507,-1.96165,-2.30278,-2.30278\}, \\
\sigma_{\mathbf{Q}}(G \bar{G})= & \{7.48933,6.09659,4.90211,4.56491,4.42094,4.17557,3.19301, \\
& 1.82443,1.7409,1.35075,1.14356,1.09789\}, \\
\sigma_{\mathbf{L}}(G \bar{G})=\{6.51053,5.90211,5.64232,5.41421,5.17557,2.82443,2.58579, & \\
& 2.35768,2.09789,2,1.48947,0\} .
\end{aligned}
$$

The determination of the roots of the polynomial $p(\rho)$ in (5) belongs to the context of nonlinear eigenvalue problems (see, for instance, $[1,10,11,14]$ ). However, in our particular case, in the next sections we take advantage from the properties of the adjacency, Laplacian and signless Laplaciam matrices of these graphs. 


\section{Adjacency eigenpairs of complementary prisms}

In this section all the eigenvalues but two for each main eigenvalue of the graph $G$ of the complementary prism $G \bar{G}$ are determined in terms of the eigenvalues of $G$. Particular attention is given when $G$ is connected and regular and in such a case all the eigenvalues of $G \bar{G}$ are determined in terms of the eigenvalues of $G$. The cases of regular and strongly regular complementary prisms are also analyzed.

\subsection{A-eigenpairs of $G \bar{G}$ in terms of the A-eigenpairs of $G$}

Non-main A-eigenvalues of $G$ determine non-main A-eigenvalues in $\bar{G}$ as asserted in the following lemma.

Lemma 3.1. [7] If $\lambda$ is an eigenvalue of $G$ with corresponding eigenvector $\mathbf{u}$ such that $\mathbf{u} \perp \mathbf{j}$, then $-1-\lambda$ is an eigenvalue of $\bar{G}$ with corresponding eigenvector u.

Theorem 3.2. If $(\lambda, \mathbf{u})$ is an eigenpair of $G$ such that $\mathbf{u} \perp \mathbf{j}$, then $\alpha_{1,2}(\lambda)=$ $\frac{-1 \pm \sqrt{(2 \lambda+1)^{2}+4}}{2}$ are eigenvalues of $G \bar{G}$ with associated eigenvectors $\mathbf{w}=$ $\left(\begin{array}{c}\mathbf{u} \\ \left(\alpha_{1,2}(\lambda)-\lambda\right) \mathbf{u}\end{array}\right)$ which are orthogonal to the all one vector.

Proof. Assuming that $\left(\begin{array}{c}\mathbf{u} \\ \beta \mathbf{u}\end{array}\right)$ is an eigenvector of $\mathbf{A}_{G \bar{G}}$ associated to some eigenvalue $\theta$ and taking into account that $\mathbf{A}_{G \bar{G}}=\left(\begin{array}{cc}\mathbf{A}_{G} & \mathbf{I} \\ \mathbf{I} & \mathbf{A}_{\bar{G}}\end{array}\right)$, it follows that

$$
\mathbf{A}_{G \bar{G}}\left(\begin{array}{c}
\mathbf{u} \\
\beta \mathbf{u}
\end{array}\right)=\left(\begin{array}{c}
\mathbf{A}_{G} \mathbf{u}+\beta \mathbf{u} \\
\mathbf{u}+\beta \mathbf{A}_{\bar{G}} \mathbf{u}
\end{array}\right)=\left(\begin{array}{c}
(\lambda+\beta) \mathbf{u} \\
(1-\beta-\beta \lambda) \mathbf{u}
\end{array}\right)=\theta\left(\begin{array}{c}
\mathbf{u} \\
\beta \mathbf{u}
\end{array}\right) .
$$

Therefore, we obtain

$\left\{\begin{array}{l}\lambda+\beta=\theta \\ 1-\beta-\beta \lambda=\theta \beta\end{array} \Leftrightarrow 1-\beta-\beta \lambda=(\lambda+\beta) \beta \Leftrightarrow \beta^{2}+(2 \lambda+1) \beta-1=0\right.$.

Thus $\beta=\frac{-(2 \lambda+1) \pm \sqrt{(2 \lambda+1)^{2}+4}}{2}=\frac{-1 \pm \sqrt{(2 \lambda+1)^{2}+4}}{2}-\lambda$ and $\theta=\frac{-1 \pm \sqrt{(2 \lambda+1)^{2}+4}}{2}$. Since $\mathbf{u} \perp \mathbf{j}$, the last part is immediate.

Remark 1. We may note that, if $\lambda$ is a non-main $\mathbf{A}$-eigenvalue of $G$ with multiplicity $m$ or a main $\mathbf{A}$-eigenvalue with multiplicity $m+1$, then there are $m$ linear independent eigenvectors $\mathbf{u}_{1}, \ldots, \mathbf{u}_{m}$ associated to $\lambda$, all orthogonal to j. As consequence of Theorem 3.2, $\alpha_{1,2}=\alpha_{1,2}(\lambda)=\frac{-1 \pm \sqrt{(2 \lambda+1)^{2}+4}}{2}$ are eigenvalues of $G \bar{G}$, each one with multiplicity at least $m$, and eigenspace orthogonal to the all one vector spanned, respectively, by the linear independent vectors

$$
\begin{aligned}
&\left(\begin{array}{c}
\mathbf{u}_{j} \\
\left(\alpha_{1}-\lambda\right) \mathbf{u}_{j}
\end{array}\right) \text { for } j=1, \ldots, m, \\
& \text { and }\left(\begin{array}{c}
\mathbf{u}_{j} \\
\left(\alpha_{2}-\lambda\right) \mathbf{u}_{j}
\end{array}\right) \text { for } j=1, \ldots, m .
\end{aligned}
$$

Additionally, $\alpha_{1}(\lambda)=-1-\alpha_{2}(\lambda)$. 
The next remark states some additional properties of the spectrum of complementary prisms.

Remark 2. If $\lambda \in\{-1,0\}$ is an eigenvalue of $G$ which is non-main or main with multiplicity greater than one, then the symmetric of golden ratio $\Phi=\frac{1+\sqrt{5}}{2}$ and $\Phi^{-1}$ are eigenvalues of the complementary prism $G \bar{G}$. In such a case, the eigenvalues $\alpha_{1,2}$ are

$$
\left\{\begin{array}{l}
\alpha_{1}(-1)=\alpha_{1}(0)=\frac{-1+\sqrt{5}}{2}=\Phi^{-1} \\
\alpha_{2}(-1)=\alpha_{2}(0)=\frac{-1-\sqrt{5}}{2}=-\Phi
\end{array}\right.
$$

However, the presence of -1 or 0 in the spectrum of $G$ is not a necessary condition for $-\Phi, \Phi^{-1} \in \sigma_{\mathbf{A}}(G \bar{G})$. For instance, $-\Phi, \Phi^{-1} \in \sigma_{\mathbf{A}}\left(P_{4} \overline{P_{4}}\right)$ and $-1,0 \notin$ $\sigma_{\mathbf{A}}\left(P_{4}\right)=\left\{ \pm \Phi, \pm \Phi^{-1}\right\}$. Furthermore, from this example, since $-\Phi, \Phi^{-1} \in$ $\sigma_{\mathbf{A}}\left(P_{4}\right) \cap \sigma_{\mathbf{A}}\left(P_{4} \overline{P_{4}}\right)$, we may conclude that there are graphs $G$ for which $\sigma_{\mathbf{A}}(G) \cap$ $\sigma_{\mathbf{A}}(G \bar{G}) \neq \emptyset$.

\subsection{Complementary prisms of connected regular graphs}

Since among $G$ and $\bar{G}$ at least one of these graphs is connected, without loss of generality we may assume that $G$ is connected.

Notice that for every graph $G$, its largest eigenvalue $\lambda_{1}$ is always a main eigenvalue. In particular, when $G$ is $r$-regular with spectrum $\lambda_{1}=r, \lambda_{2}, \ldots, \lambda_{n}$, all eigenvalues but $\lambda_{1}$ are non-main [3]. For the spectrum of the complementary prism of a connected regular graph $G$ we have the following result.

Theorem 3.3. If $G$ is a connected $k$-regular graph on $n$ vertices, then

$$
\beta_{1,2}=\beta_{1,2}(n, k)=\frac{(n-1) \pm \sqrt{(n-1-2 k)^{2}+4}}{2}
$$

are eigenvalues of $G \bar{G}$ with corresponding eigenvectors

$$
\mathbf{v}_{i}=\left(\begin{array}{c}
\mathbf{j} \\
\left(\beta_{i}-k\right) \mathbf{j}
\end{array}\right) \text {, for } i=1,2 \text {, respectively }
$$

If $G \bar{G}$ is non regular, then it is biregular and the eigenvalues (8) are the two main eigenvalues of $\bar{G} \bar{G}$.

Proof. The proof is similar to the proof of Theorem 3.2.

As an immediate consequence of Theorems 3.2 and 3.3, we may determine the all spectrum of the complementary prism $G \bar{G}$ of a $k$-regular graph $G$ of order $n$ in terms of $n$ and the spectrum of $G$, as the following corollary states.

Corollary 3.4. If $G$ is a connected $k$-regular graph on $n$ vertices with spectrum $\sigma_{\mathbf{A}}=\left\{\gamma_{1}^{\left[m_{1}\right]}, \ldots, \gamma_{p}^{\left[m_{p}\right]}\right\}$, then the eigenvalues of $G \bar{G}$ are

$$
\begin{aligned}
& \text { (1) } \alpha_{1, i}\left(\gamma_{i}\right)=\frac{-1+\sqrt{\left(2 \gamma_{i}+1\right)^{2}+4}}{2} \text {, with multiplicity } m_{i} \text {, for } 1 \leq i \leq p \text {; } \\
& \text { (2) } \alpha_{2, i}\left(\gamma_{i}\right)=\frac{-1-\sqrt{\left(2 \gamma_{i}+1\right)^{2}+4}}{2} \text {, with multiplicity } m_{i} \text {, for } 1 \leq i \leq p
\end{aligned}
$$


(3) $\beta_{1,2}(n, k)=\frac{n-1 \pm \sqrt{(n-1-2 k)^{2}+4}}{2}$, which are the two main eigenvalues when $G \bar{G}$ is non regular.

Example 3.5. Let us determine the spectra of the complementary prisms of the complete graphs $K_{n}$, which are the coronas $K_{n} \circ K_{1}$, the complementary prisms of the complete bipartite graphs $K_{n, n}$ and the complementary prism of the Petersen graph $C_{5} \overline{C_{5}}$.

1. Since $\sigma_{\mathbf{A}}\left(K_{n}\right)=\left\{-1^{[n-1]}, n-1\right\}$, setting $\mathcal{G}_{n}=K_{n} \overline{K_{n}}$, the spectra of the graphs of the infinite family $\left(\mathcal{G}_{n}\right)_{n \geq 2}$ have only the following four distinct eigenvalues

$$
\sigma_{\mathbf{A}}\left(\mathcal{G}_{n}\right)=\left\{(-\Phi)^{[n-1]},\left(\Phi^{-1}\right)^{[n-1]}, \frac{n-1 \pm \sqrt{(n-1)^{2}+4}}{2}\right\}
$$

2. Since $\sigma_{\mathbf{A}}\left(K_{n, n}\right)=\left\{n, 0^{[2 n-2]},-n\right\}$, setting $\mathcal{H}_{n}=K_{n, n} \overline{K_{n, n}}$, the graphs of the infinite family $\left(\mathcal{H}_{n}\right)_{n \geq 2}$ have only the following six distinct eigenvalues

$$
\sigma_{\mathbf{A}}\left(\mathcal{H}_{n}\right)=\left\{(-\Phi)^{[2 n-2]},\left(\Phi^{-1}\right)^{[2 n-2]}, n-\Phi, n+\Phi^{-1}, \frac{-1 \pm \sqrt{(2 n-1)^{2}+4}}{2}\right\} .
$$

3. Since $\sigma_{\mathbf{A}}\left(C_{5} \overline{C_{5}}\right)=\left\{3,1^{[5]},(-2)^{[4]}\right\}$, we have

$$
\sigma_{\mathbf{A}}\left(\left(C_{5} \overline{C_{5}}\right) \overline{\left(C_{5} \overline{C_{5}}\right)}\right)=\left\{\frac{9 \pm \sqrt{13}}{2},\left(\frac{-1+\sqrt{13}}{2}\right)^{[9]},\left(\frac{-1-\sqrt{13}}{2}\right)^{[9]}\right\}
$$

We may recall that a graph of order $n$ is called strongly regular with parameters $(n, r, a, b)$ whenever it is not complete or edgeless, each vertex is adjacent to $r$ vertices, for each pair of adjacent vertices there are $a$ vertices adjacent to both and for each pair of non-adjacent vertices there are $b$ vertices adjacent to both. A connected regular graph is strongly regular if and only if it has exactly three distinct eigenvalues [3, Th. 3.32]. The next theorem states a couple of properties of regular complementary prisms. Namely, it states that there is only one strongly regular graph, $C_{5}$, whose complementary prism, $C_{5} \overline{C_{5}}$ (the Petersen graph), is strongly regular.

Theorem 3.6. Let $G$ be a connected graph on $n>3$ vertices.

1. $G \bar{G}$ is regular if and only if $G$ is $k$-regular with $k=\frac{n-1}{2}$.

2. $G \bar{G}$ is strongly regular if and only if $G=C_{5}$.

Proof. Let us prove each of the statements.

1. It is immediate that when $G$ is not regular $G \bar{G}$ is not regular as well. Thus, assuming that $G$ is $k$-regular, it follows that each vertex $v \in V(G \bar{G}) \cap V(G)$ has degree $k+1$ and each vertex $v^{\prime} \in V(G \bar{G}) \cap V(\bar{G})$ has degree $n-k$. Therefore, $G \bar{G}$ is regular if and only if $k+1=n-k$. 
2. Assume that $G \bar{G}$ is a strongly regular graph. According to the previous statement, $G$ is a $k$-regular graph with $k=\frac{n-1}{2}$. Therefore, by Corollary 3.4-(3), $r=\beta_{1}=\frac{n+1}{2}$ and $\beta_{2}=\frac{n-3}{2}$ are eigenvalues of $G \bar{G}$.

Let $\frac{n-1}{2}>\lambda_{2} \geq \cdots \geq \lambda_{n}$ be the eigenvalues of $G$. Taking into account that $G \bar{G}$ has just three distinct eigenvalues, from Corollary 3.4 (1) and (2) it follows that

$$
\begin{aligned}
& -1+\sqrt{\left(2 \lambda_{2}+1\right)^{2}+4}=\cdots=-1+\sqrt{\left(2 \lambda_{n}+1\right)^{2}+4}=n-3, \\
& -1-\sqrt{\left(2 \lambda_{2}+1\right)^{2}+4}=\cdots=-1-\sqrt{\left(2 \lambda_{n}+1\right)^{2}+4}
\end{aligned}
$$

Equations (10) imply $\lambda_{j}=\frac{-1 \pm \sqrt{n(n-4)}}{2}, 2 \leq j \leq n$. Therefore, $G$ is a regular graph with three distinct eigenvalues, namely, $k=\frac{n-1}{2}$,

$$
\begin{aligned}
& \gamma_{1}=\frac{-1+\sqrt{n(n-4)+4}}{2} \text { and } \\
& \gamma_{2}=\frac{-1-\sqrt{n(n-4)+4}}{2}
\end{aligned}
$$

and thus $G$ is strongly regular. Let us suppose that $\left(n, \frac{n-1}{2}, x, y\right)$ are the parameters of $G$. Then, as it is well known, from the parameters it follows that the distinct eigenvalues of $G$ are $k=\frac{n-1}{2}$,

$$
\begin{aligned}
\gamma_{1} & =\frac{x-y+\sqrt{(x-y)^{2}+4(k-y)}}{2}, \\
\text { and } \gamma_{2} & =\frac{x-y-\sqrt{(x-y)^{2}+4(k-y)}}{2}
\end{aligned}
$$

From (11) - (13) and (12) - (14) we obtain the equations

$$
\begin{aligned}
& \frac{x-y+\sqrt{(x-y)^{2}+4(k-y)}}{2}=\frac{-1+\sqrt{n(n-4)}}{2} \\
& \frac{x-y-\sqrt{(x-y)^{2}+4(k-y)}}{2}=\frac{-1-\sqrt{n(n-4)}}{2}
\end{aligned}
$$

and (15) and (16) imply $x-y=-1$. Therefore

$$
1+4\left(\frac{n-1}{2}-y\right)=n(n-4) \Leftrightarrow n(6-n)=4 y+1 .
$$

Since $y \geq 0$, then $n \leq 5$. As $C_{5}$ is the unique non complete regular graph of odd order not greater than 5 , the result follows.

The converse is immediate, taking into account that $C_{5}$ is the strongly regular graph with parameters $(5,2,0,1)$ and its complementary prism is the Petersen graph. 


\section{Signless Laplacian eigenpairs of complemen- tary prisms}

The results of this section are mostly similar to those obtained in Subsections 3.1 and 3.2. From (1) it follows that

$$
\mathbf{Q}_{G \bar{G}}=\left(\begin{array}{cc}
\mathbf{Q}_{G}+\mathbf{I} & \mathbf{I} \\
\mathbf{I} & \mathbf{Q}_{\bar{G}}+\mathbf{I}
\end{array}\right)=\left(\begin{array}{cc}
\mathbf{Q}_{G} & \mathbf{0} \\
\mathbf{0} & \mathbf{Q}_{\bar{G}}
\end{array}\right)+\left(\begin{array}{cc}
\mathbf{I} & \mathbf{I} \\
\mathbf{I} & \mathbf{I}
\end{array}\right) .
$$

Lemma 4.1. Let $G$ be a graph of order $n$. If $(q, \mathbf{u})$ is a $\mathbf{Q}$-eigenpair of $G$ such that $\mathbf{u} \perp \mathbf{j}$, then $(n-2-q, \mathbf{u})$ is a $\mathbf{Q}$-eigenpair of $\bar{G}$.

Proof. Notice that

$$
\mathbf{Q}_{\bar{G}}=\mathbf{Q}_{K_{n}}-\mathbf{Q}_{G} .
$$

Since $K_{n}$ is regular with $\sigma_{\mathbf{Q}}\left(K_{n}\right)=\left\{2(n-1),(n-2)^{[n-1]}\right\}$, its unique main $\mathbf{Q}$-eigenvalue is $2(n-1)$ (see [5]). From $\mathbf{u} \perp \mathbf{j}$, it follows that $\mathbf{Q}_{K_{n}} \mathbf{u}=(n-2) \mathbf{u}$ and then

$$
\begin{aligned}
\mathbf{Q}_{\bar{G}} \mathbf{u} & =\mathbf{Q}_{K_{n}} \mathbf{u}-\mathbf{Q}_{G} \mathbf{u} \\
& =(n-2-q) \mathbf{u}
\end{aligned}
$$

In the next result all the Q-eigenvalues of the complementary prism $G \bar{G}$ but two for each main $\mathbf{Q}$-eigenvalue of the graph $G$ are determined.

Theorem 4.2. Let $G$ be a graph of order $n$. If $(q, \mathbf{u})$ is a $\mathbf{Q}$-eigenpair of $G$ such that $\mathbf{u} \perp \mathbf{j}$, then $\rho_{1,2}(q)=\frac{n \pm \sqrt{(n-2(q+1))^{2}+4}}{2}$ are $\mathbf{Q}$-eigenvalues of $G \bar{G}$ with associated eigenvectors $\left(\begin{array}{c}\mathbf{u} \\ \left(\rho_{1,2}(q)-(q+1)\right) \mathbf{u}\end{array}\right)$ which are orthogonal to the all one vector.

Proof. This proof is similar to the proof of Theorem 3.2.

When $G$ is a connected regular graph the following results are obtained using a technique similar to the one used in Section 3.2.

Theorem 4.3. If $G$ is a connected $k$-regular graph on $n$ vertices, then

$$
\varrho_{1,2}=\varrho_{1,2}(n, k)=n \pm \sqrt{(n-2 k-1)^{2}+1}
$$

are Q-eigenvalues of $G \bar{G}$ with associated eigenvectors $\left(\begin{array}{c}\mathbf{j} \\ \left(\varrho_{1,2}-2 k-1\right) \mathbf{j}\end{array}\right)$. These eigenvalues are the two main $\mathbf{Q}$-eigenvalues of $G \bar{G}$ when this graph is non regular.

Proof. The proof is similar to the one of Theorem 4.2, taking into account that when $G$ is connected and regular all its $\mathbf{Q}$-eigenvalues are non main, except the largest one $([5])$.

The next corollary is just a signless Laplacian version of Corollary 3.4. 
Corollary 4.4. If $G$ is a connected $k$-regular graph on $n$ vertices such that $\sigma_{\mathbf{Q}}=\left\{\pi_{1}^{\left[t_{1}\right]}, \ldots, \pi_{p}^{\left[t_{p}\right]}\right\}$, then the $\mathbf{Q}$-eigenvalues of $G \bar{G}$ are

(1) $\rho_{1, i}\left(\pi_{i}\right)=\frac{n+\sqrt{\left(n-2\left(\pi_{i}+1\right)\right)^{2}+4}}{2}$, with multiplicity $t_{i}$, for $1 \leq i \leq p$;

(2) $\rho_{2, i}\left(\pi_{i}\right)=\frac{n-\sqrt{\left(n-2\left(\pi_{i}+1\right)\right)^{2}+4}}{2}$, with multiplicity $t_{i}$, for $1 \leq i \leq p$;

(3) $\varrho_{1,2}(n, k)=n \pm \sqrt{(n-2 k-1)^{2}+1}$, which are the two main $\mathbf{Q}$-eigenvalues obtained when $G \bar{G}$ is non regular.

\section{$5 \quad$ Laplacian eigenpairs of complementary prisms}

The following well known lemma establishes that the Laplacian spectrum of $\bar{G}$ is closely related to the Laplacian spectrum of $G$.

Lemma 5.1. If the Laplacian eigenvalues of $G$ are $\mu_{1} \geq \mu_{2} \geq \cdots \geq \mu_{n-1} \geq$ $\mu_{n}=0$, then the Laplacian eigenvalues of $\bar{G}$ are $n-\mu_{n-1} \geq \cdots \geq n-\mu_{2} \geq$ $n-\mu_{1} \geq 0$, and for every $i \in\{1, \ldots, n-1\}$ the Laplacian eigenvalue $\mu_{i}$ of $G$ and the Laplacian eigenvalue $n-\mu_{i}$ have the same associated eigenspace.

As we may note, it follows from (1) that

$$
\mathbf{L}_{G \bar{G}}=\left(\begin{array}{cc}
\mathbf{L}_{G}+\mathbf{I} & -\mathbf{I} \\
-\mathbf{I} & \mathbf{L}_{\bar{G}}+\mathbf{I}
\end{array}\right) .
$$

Theorem 5.2. Let $G$ be graph on $n$ vertices with Laplacian eigenvalues $\mu_{1} \geq$ $\mu_{2} \geq \cdots \geq \mu_{n-1} \geq \mu_{n}=0$. For each $i=1, \ldots, n-1$, if $\left(\mu_{i}, \mathbf{u}_{i}\right)$, is a $\mathbf{L}$-eigenpair of $G$ then

$$
\tau_{1,2}\left(\mu_{i}\right)=\frac{n+2 \pm \sqrt{\left(n-2 \mu_{i}\right)^{2}+4}}{2}
$$

are $\mathbf{L}$-eigenvalues of $G \bar{G}$ with associated eigenvectors $\left(\begin{array}{c}\mathbf{u}_{i} \\ \left(\mu_{i}-\tau_{1,2}\left(\mu_{i}\right)\right) \mathbf{u}_{i}\end{array}\right)$. The others $\mathbf{L}$-eigenvalues of $G \bar{G}$ are 2 and 0 with associated eigenvectors $\left(\begin{array}{r}\mathbf{j} \\ -\mathbf{j}\end{array}\right)$ and $\left(\begin{array}{l}\mathbf{j} \\ \mathbf{j}\end{array}\right)$, respectively.

Proof. Let $i \in\{1, \ldots, n-1\}$ and assume that $\mathbf{w}_{i}=\left(\begin{array}{c}\mathbf{u}_{i} \\ \beta_{i} \mathbf{u}_{i}\end{array}\right)$ is an eigenvector of $\mathbf{L}_{G \bar{G}}$ associated to the eigenvalue $\tau_{i}$. Then, taking into account (18) and Lemma 5.1, from the eigenvector equation $\mathbf{L}_{G \bar{G}} \mathbf{w}_{i}=\tau_{i} \mathbf{w}_{i}$ it follows that

$$
\begin{aligned}
& \left(\begin{array}{r}
\left(\mu_{i}+1-\beta_{i}\right) \mathbf{u}_{i} \\
\left(-1+\left(n-\mu_{i}+1\right) \beta_{i}\right) \mathbf{u}_{i}
\end{array}\right)=\tau_{i}\left(\begin{array}{r}
\mathbf{u}_{i} \\
\beta_{i} \mathbf{u}_{i}
\end{array}\right) \text {. So, } \\
& \left\{\begin{array}{lll}
\mu_{i}+1-\beta_{i} & =\tau_{i} \\
-1+\left(n-\mu_{i}+1\right) \beta_{i} & = & \tau_{i} \beta,
\end{array}\right.
\end{aligned}
$$

which implies the quadratic equation

$$
\beta_{i}^{2}-\left(2 \mu_{i}-n\right) \beta_{i}-1=0 .
$$


Therefore, $\beta_{i}=\frac{2 \mu_{i}-n \pm \sqrt{\left(2 \mu_{i}-n\right)^{2}+4}}{2}$ and from (19) we obtain

$$
\tau_{1,2}\left(\mu_{i}\right)=\frac{n+2 \pm \sqrt{\left(2 \mu_{i}-n\right)^{2}+4}}{2} .
$$

The last part is immediate.

Example 5.3. Let us apply Theorem 5.2 to compute all the Laplacian eigenvalues of $G \bar{G}$, where $G$ is the graph of Example 2.3. Taking into account that $\sigma_{\mathbf{L}}(G)=\left\{\frac{7+\sqrt{5}}{2}, \frac{5+\sqrt{13}}{2}, \frac{7-\sqrt{5}}{2}, 2, \frac{5-\sqrt{13}}{2}, 0\right\}$, the $\mathbf{L}$-eigenvalues are 2,0 and the ones presented in the following table.

\begin{tabular}{|c|c|c|}
\hline$\mu$ & $\tau_{1}(\mu)$ & $\tau_{2}(\mu)$ \\
\hline$\frac{7+\sqrt{5}}{2}$ & $\frac{8+\sqrt{10+2 \sqrt{5}}}{2}$ & $\frac{8-\sqrt{10+2 \sqrt{5}}}{2}$ \\
$\frac{5+\sqrt{13}}{2}$ & $\frac{8+\sqrt{18-2 \sqrt{13}}}{2}$ & $\frac{8-\sqrt{18-2 \sqrt{13}}}{2}$ \\
$\frac{7-\sqrt{5}}{2}$ & $\frac{8+\sqrt{10-2 \sqrt{5}}}{2}$ & $\frac{8-\sqrt{10-2 \sqrt{5}}}{2}$ \\
2 & $4+\sqrt{2}$ & $4-\sqrt{2}$ \\
$\frac{5-\sqrt{13}}{2}$ & $\frac{8+\sqrt{18+2 \sqrt{13}}}{2}$ & $\frac{8-\sqrt{18+2 \sqrt{13}}}{2}$ \\
\hline
\end{tabular}

Corollary 5.4. If $G$ is a graph on $n$ vertices with Laplacian eigenvalues $\mu_{1}$, $\mu_{2}, \ldots, \mu_{n-1}$ and $\mu_{n}=0$, then the characteristic polynomial of the Laplacian matrix of $G \bar{G}$ is

$$
p(\tau)=\operatorname{det}\left(\tau \mathbf{I}_{2 n}-\mathbf{L}_{G \bar{G}}\right)=\tau(\tau-2) \prod_{i=1}^{n-1}\left(\tau^{2}-(n+2) \tau+n\left(\mu_{i}+1\right)-\mu_{i}{ }^{2}\right) .
$$

\section{Conclusions and open problems}

The Petersen graph $C_{5} \overline{C_{5}}$ and $K_{n} \overline{K_{n}}$, the corona of the complete graph $K_{n}$ and $K_{1}$, are examples of complementary prisms of graphs. As we have proven, the Petersen graph is the unique complementary prism which is strongly regular. The Corollary 2.2 presents the $\mathbf{A}, \mathbf{Q}$ and $\mathbf{L}$-eigenvalues of complementary prisms $G \bar{G}$ as roots of the polynomial

$$
p(\rho)=\operatorname{det}\left(\left(\mathbf{M}_{G}-(\rho-\delta) \mathbf{I}\right)\left(\mathbf{M}_{\bar{G}}-(\rho-\delta) \mathbf{I}\right)-\mathbf{I}\right),
$$

where $\mathbf{M} \in\{\mathbf{A}, \mathbf{Q}, \mathbf{L}\}$ and $\delta= \begin{cases}0, & \text { if } \mathbf{M}=\mathbf{A} \text {; } \\ 1, & \text { otherwise. }\end{cases}$

All the eigenpairs of the matrix $\mathbf{M} \in\left\{\mathbf{A}_{H}, \mathbf{Q}_{H}, \mathbf{L}_{H}\right\}$ representing the complementary prism $H=G \bar{G}$ of the graph $G$ are computed in terms of the eigenvalues of the corresponding matrix $\mathbf{M} \in\left\{\mathbf{A}_{G}, \mathbf{Q}_{G}, \mathbf{L}_{G}\right\}$, with exception of two of them for each main eigenvalue of $\mathbf{A}_{G}$ and $\mathbf{Q}_{G}$, respectively. Particular attention was given to the complementary prisms of regular graphs. From the obtained results it is immediate that if the matrices $\mathbf{M}_{G}$ and $\mathbf{M}_{H}$ representing two graphs $G$ and $H$ are cospectral, then $\mathbf{M}_{G \bar{G}}$ and $\mathbf{M}_{H \bar{H}}$ are cospectral when $\mathbf{M}=\mathbf{L}$ and also when $\mathbf{M} \in\{\mathbf{A}, \mathbf{Q}\}$ and $G$ and $H$ are connected regular graphs.

Among several unexplored research directions on the topics of this paper, so far, the following problems remain unsolved. 
1. Can we compute all the $\mathbf{M}$-eigenvalues of the complementary prism $G \bar{G}$ in terms of the $\mathbf{M}$-eigenvalues of $G$, when $\mathbf{M} \in\{\mathbf{A}, \mathbf{Q}\}$ ?

2. Can we decide wether a graph $H$ is a complementary prism of a graph from the spectrum of $\mathbf{M}_{H}$, for $\mathbf{M} \in\{\mathbf{A}, \mathbf{Q}, \mathbf{L}\}$ ?

3. In this paper it is proven that there is only one regular complementary prism with three distinct eigenvalues. Are there non regular complementary prisms with just three distinct eigenvalues?

\section{Acknowledgement}

The research of D.M. Cardoso and P. Carvalho was partially supported by the Portuguese Foundation for Science and Technology ("FCT-Fundação para a Ciência e a Tecnologia"), through the CIDMA - Center for Research and Development in Mathematics and Applications, within project UID/MAT/04106/2013. These authors also thanks the hospitality of COPPE of UFRJ, where part of this research was conducted. M.A.A. de Freitas was partially supported by National Council of Technological and Scientific Development(CNPq-Conselho Nacional de Desenvolvimento Científico e Tecnológico) and FAPERJ (Fundação de Amparo à Pesquisa do Estado do Rio de Janeiro). C.T.M.Vinagre thanks the hospitality of University of Aveiro, where this research was started.

\section{References}

[1] W.E. ARNOLDI. The principle of minimized iterations in the solution of the matrix eigenvalue problem. Q. Appl. Math. 9 (1951): 17-29.

[2] A. E. Brouwer, W. H. Haemers. Spectra of Graphs. Springer, New York, 2012.

[3] D. Cvetković, M. Doob, H. SAchs. Spectra of Graphs - Theory and Application. Johann Ambrosius Barth Verlag, Heidelberg-Leipzig, 1995.

[4] D. Cvetković, P. Rowlinson S.K. Simić. An Introduction to the Theory of Graph Spectra. Cambridge University Press, Cambridge, 2010.

[5] H. Deng, H. Huang. On the main signless Laplacian eigenvalues of a graph. Electronic Journal of Linear Algebra 26 (2013).

[6] M. A. Duarte, L. Penso, D. Rautenbach, U.S. Souza. Complexity properties of complementary prisms. J. Comb. Optim 33 (2017):365-372.

[7] E.M. Hagos. Some results on graph spectra Linear Algebra Appl., 356 (2002), pp. 103-111.

[8] T. W. Haynes, M. A. Hennng, P J. Slater, L. C. van der Merwe. The complementary product of two graphs. Bulletin of the ICA 51 (2007): 21-30.

[9] T. W. Haynes, M. A. Henning, L. C. van der Merwe. Domination and total domination in complementary prisms. J. Comb. Optim 18(1) (2009): 23-37.

[10] E. Jarlebring, W. Michiels, K. Meerbergen. A linear eigenvalue algorithm for the nonlinear eigenvalue problem. Numer. Math. 122 (2012): 169-195. 
[11] V. Mehrmann, H. Voss. Nonlinear eigenvalue problems: a challenge for modern eigenvalue methods. GAMM Mitteilungen 27 (2004): 121-152.

[12] D. Meierling, F. Protti, D. Rautenbach, A. R. Almeida. Cycles in complementary prisms. Discrete Applied Mathematics. 193 (2015): 180-186.

[13] H. Shapiro. Linear Algebra and Matrices - topics for a second course. Pure and Applied Undergraduate Texts 24, AMS, Providence, 2015.

[14] H. Voss. An Arnoldi method for nonlinear eigenvalue problems. BIT Numerical Mathematics 44 (2004): 387-401. 